\title{
General Description of Dendrite Growth in Unidirectional Solidification of Alloys*
}

\author{
By Yasunori MIYATA** and Toshio SUZUKI**
}

\begin{abstract}
Synopsis
General shapes of the dendritic interface are described in the paper and a perturbation theory for the dendrite growth on the interface is proposed. The radius of the curvature predicted for the elliptic tip slightly deviates from that for the parabolic one of revolution, but the temperature of elliptic tip predicted deviates from that of the palabolic one. This shows that the elliptic tip gives a rather good approximation for the shape of dendrite tip with respect to the dimensions. The application of the theory to the primary arm spacing is also given.
\end{abstract}

\section{Introduction}

Recently, several analytical models have been proposed to explain the structure of dendrite in unidirectional solidification. However, in these models, the shape of dendrite tip is usually assumed to be hemispherical or parabolic, in order to simplify the problem. Although the tip temperature and concentration of dendrite tip can fairly well be predicted, radius of curvature predicted is different according to the model adopted. Therefore, the primary arm spacing predicted is different.

The theories mentioned above have been derived by adopting the assumptions about the stability of tip. Kurz and Fisher ${ }^{1)}$ used the critical wave-length for the tip radius given by the perturbation theory for planar interface. ${ }^{2)}$ Burden and Hunt ${ }^{3)}$ assumed the maximum growth rate. On the other hand, Trivedi $^{4}$ ) used the perturbative stability condition ${ }^{5)}$ for the hemispherical tip. These are the origin for the different predictions for dimensions of dendrite. The authors proposed a perturbation theory ${ }^{6}$ ) of cellular and dendritic growth for a tip of a paraboloid of revolution.

The shapes of tip experimentally observed for metals are usually hemispherical for cells and a paraboloid of revolution for dendrites. On the other hand, for the organic system with small thermal diffusivity, the shape of dendrite tip experimentally observed is usually parabolic, because of larger region of dendrite tip. Accordingly, if the paraboloid of revolution is assumed, it is possible to approximate the shape of dendrite tip found in organic systems. However, in the case of metal, the region of dendrite tip is smaller than those of organic systems. Therefore, the assumption of the paraboloid of revolution does not give a good approximation for the shape of dendrite tip of metal. The influence of the shape of tip assumed on the dendrite one has not been acquired in the previous models. The aim of this study is to clarify the effects of the shape of dendrite on the radius of dendrite tip curvature, the primary arm spacing and the tip concentration of solute (or tip temperature).

\section{Dendrite with Quadratic Interface}

\section{Dendrite}

To describe the shape of dendrite, it is possible to take the dimensionless parabolic coordinates $(\xi, \eta, \phi)$, which are related to the following dimensionless Cartesian coordinates $(x, y, z)$.

$$
x=\xi \eta \cos \phi, \quad y=\xi \eta \sin \phi, z=\frac{1}{2}\left(\xi^{2}-\eta^{2}\right)
$$

In this coordinate system, a dendrite with the interface of a paraboloid of revolution is given as

$$
\xi_{0}^{2}=1
$$

Let us now consider the interface given by the following equation,

$$
\xi_{0}^{2}=1+a\left(\eta^{2}-1\right)
$$

where, $a$ : constant parameter.

If the dimensionless Cartesian coordinate is adopted, this interface can be expressed by Eq. (3).

$$
\frac{x^{2}+y^{2}}{\left[-\frac{(1-a)^{2}}{4 a}\right]}+\frac{\left[z-\frac{1-a^{2}}{4 a}\right]^{2}}{\left[\frac{(1-a)^{2}}{4 a}\right]^{2}}=1
$$

From Eq. (3), shapes of interface can easily be obtained. They are classified into

$$
\left.\begin{array}{ll}
a=1 & \text { for planar interface } \\
0<a<1 & \text { for hyperbolic interface } \\
a=0 & \text { for parabolic interface } \\
-1<a<0 & \text { for elliptic interface } \\
a=-1 & \text { for hemispherical interface }
\end{array}\right)
$$

It can be seen that the coordinates are normalized by the radius of tip curvature. Therefore, it is considered that the dendritic interface for quadratic shape can be expressed by Eq. (2).

Next problem is how to construct the perturbation model for a dendrite with quadratic interface.

\footnotetext{
* Based on the paper presented to the 110th ISIJ Meeting, October 1985, S958, at Niigata University, in Niigata. Manuscript received on April 22, 1986; accepted in the final form on July 4, 1986. (C) 1986 ISIJ

** Faculty of Engineering, The Technological University of Nagaoka, Kamitomioka-cho, Nagaoka 940-21.
} 


\section{Perturbation Theory and Prediction of the Radius of Curvature}

The temperature of liquid, the temperature of solid and the concentration of solute in liquid are given as the solutions ${ }^{6}$ ) of the diffusion equations in the coordinate system $(\xi, \eta, \phi)$

$$
\begin{aligned}
& \mathcal{T}_{L}^{(0)}=T_{0}^{L}+A_{0}^{L} E_{1}\left(p_{L} \xi^{2}\right)+B_{0}^{L} e^{-p_{L} \hat{\xi}^{2}} e^{p_{L} \eta^{2}} \\
& \mathcal{T}_{S}^{(0)}=\mathcal{T}_{0}^{S}+B_{0}^{S} e^{-p_{S} \xi^{2}} e^{p_{S} \eta^{2}} \ldots \ldots \ldots \ldots \ldots \ldots \ldots \ldots \ldots \ldots \ldots \ldots \ldots \ldots \ldots \\
& C_{L}^{(0)}=C_{\infty}^{L}+A_{0} E_{1}\left(p \xi^{2}\right)+B_{0} e^{-p \xi^{2}} e^{p \eta^{2}} \ldots \ldots \ldots \ldots
\end{aligned}
$$

where, $T_{0}^{L}, A_{0}^{L}, B_{0}^{L}, T_{0}^{S}, B_{0}^{S}, A_{0}, B_{0}$ : constants to be determined from the boundary conditions

$p_{L}, p_{S}, p$ : Peclet numbers of respective temperature and solute concentration.

In this case, the solute diffusion in the solid is neglected.

The perturbed fields are given

$$
\begin{aligned}
& T_{L}=T_{L}^{(0)}\left(\xi^{2}=\xi_{0}^{2}+\Delta \xi^{2}\right)+\Delta \xi^{2}\left[\tilde{A}_{L} \widetilde{\Psi}_{n m}\left(p_{L} \xi^{2}\right) L_{n}^{(m)}\left(p_{L} \eta^{2}\right)\right. \\
& \left.+\tilde{B}_{L} \tilde{L}_{n}^{(m)}\left(p_{L} \xi^{2}\right) \Phi_{n m}\left(p_{L} \eta^{2}\right)\right] \\
& T_{S}=T_{S}^{(0)}\left(\xi^{2}=\xi_{0}^{2}+\Delta \xi^{2}\right)+\Delta \xi^{2} \tilde{L}_{n}^{(m)}\left(p_{S} \xi^{2}\right) \Phi_{n m}\left(p \eta^{2}\right)
\end{aligned}
$$

$$
\begin{aligned}
C_{L}= & C_{L}^{(0)}\left(\xi^{2}=\xi_{0}^{2}+\Delta \xi^{2}\right)+\Delta \xi^{2}\left[\tilde{A} \tilde{\Psi}_{n m}\left(p \xi^{2}\right) L_{n}^{(m)}\left(p_{S} \eta^{2}\right)\right. \\
& \left.+\tilde{B} \tilde{L}_{n}^{(m)}\left(p \xi^{2}\right) \Phi_{n m}\left(p \eta^{2}\right)\right] \ldots \ldots \ldots \ldots \ldots \ldots \ldots \ldots \ldots \ldots \ldots \ldots \ldots \ldots \ldots \ldots \ldots \ldots \ldots
\end{aligned}
$$

where, $\tilde{A}_{L}, \tilde{B}_{L}, \tilde{B}_{S}, \tilde{A}, \tilde{B}$ : parameters to be determined.

The boundary conditions for the unperturbed fields are determined on Eq. (2). The perturbed interface becomes as $\xi^{2}=\xi_{0}^{2}+\Delta \xi^{2}$, where $\Delta \xi^{2}=\delta_{n m} \sin (\mathrm{m} \phi) \cdot\left(\eta^{2}\right)^{m / 2}$ or $\Delta \xi^{2}=\delta_{n m} \cos (\mathrm{m} \phi) \cdot\left(\eta^{2}\right)^{m / 2}$.

If the boundary conditions are calculated by the same manner as that used by Miyata and Suzuki, ${ }^{6)}$ the boundary conditions show the linear relations for the parameters, $T_{0}^{L}, A_{0}^{L}, B_{0}^{L}, T_{0}^{S}, B_{0}^{s}, A_{0}, B_{0}, \tilde{A}_{L}, \tilde{B}_{L}$, $\tilde{B_{S}}, \tilde{A}$ and $\tilde{B}$. Since the behaviours of the fields near the tip are important, the fields are expanded in powers of $\eta^{2}$ and are required to satisfy the boundary conditions.

For the unperturbed fields:

From the continuity of temperature across the interface, Eqs. (9) and (10) are obtained.

$$
\begin{aligned}
& r^{0}: \quad T_{0}^{L}+A_{0}^{L} E_{1}\left(p_{L}(1-a)\right)+B_{0}^{L} e^{-p_{L}(1-a)} \\
& =T_{0}^{S}+B_{0}^{S} e^{-p_{S}(1-a)} \\
& \eta^{2}: \quad-\frac{a}{1-a} A_{0}^{L} e^{-p_{L}(1-a)}+(1-a) p_{L} B_{0}^{L} e^{-p_{L}(1-a)} \\
& =(1-a) p_{S} B_{0}^{S} e^{-p_{S}(1-a)}
\end{aligned}
$$

If the balance of heat flux at the tip is taken into account, it can be expressed as follows:

$$
\begin{aligned}
p_{L} \frac{\Delta H}{c_{1}}= & -\left[-\frac{1}{1-a} A_{0}^{L} e^{-p_{L}(1-a)}-p_{L} B_{0}^{L} e^{-p_{L}(1-a)}\right] \\
& +\frac{\kappa_{S}}{K_{L}}\left[-p_{S} B_{0}^{S} e^{-p_{S}(1-a)}\right] \ldots \ldots \ldots \ldots \ldots \ldots \ldots \ldots \ldots \ldots \ldots \ldots
\end{aligned}
$$

Introducing the temperature gradient at the tip in liquid, Eq. (12) is obtained.

$$
G_{L}=\frac{2}{\rho}\left[-\frac{1}{1-a} A_{0}^{L} e^{-p_{L}(1-a)}-p_{L} B_{0}^{L} e^{-p_{L}(1-a)}\right]
$$

From the mass flux balance of solute at the tip, it can be written as

$$
\begin{array}{r}
p(1-k)\left[C_{\infty}^{L}+A_{0} E_{1}(p(1-a))+B_{0} e^{-p(1-a)}\right] \\
\quad=-\left[-\frac{1}{1-a} A_{0} e^{-p(1-a)}-p B_{0} e^{-p(1-a)}\right]
\end{array}
$$

If the relation between temperature and solute at the interface is introduced, Eqs. (14) and (15) are obtained.

$$
\begin{gathered}
\eta^{0}: \quad T_{0}^{S}+B_{0}^{S} e^{-p_{S}(1-a)}=T_{M}-\frac{\gamma}{\Delta S} \frac{2}{\rho} \\
+m_{0}\left[C_{\infty}^{L}+A_{0} E_{1}(p(1-a))+B_{0} e^{-p(1-a)}\right] \\
\ldots \ldots \ldots \ldots \ldots \ldots \ldots \ldots \ldots \ldots \ldots \ldots \ldots \ldots \\
\eta^{2}: \quad(1-a) p_{S} B_{0}^{S} e^{-p_{S}(1-a)}=(1-a) \frac{\gamma}{\Delta S} \frac{2}{\rho} \\
+m_{0}\left[-\frac{a}{1-a} A_{0} e^{-p(1-a)}+(1-a) p B_{0} e^{-p(1-a)}\right]
\end{gathered}
$$

For the perturbed fields:

From the continuity of temperature on the interface, the following equations are obtained.

$$
\begin{aligned}
& r^{0}: \quad-\frac{1}{1-a} A_{0}^{L} e^{-p_{L}(1-a)}-p_{L} B_{0}^{L} e^{-p_{L}(1-a)} \\
& +\tilde{A}_{L} \tilde{T}_{n m}\left(p_{L}(1-a)\right) L_{n}^{(m)}(0)+\tilde{B}_{L} \tilde{L}_{n}^{(m)}\left(p_{L}(1-a)\right) \\
& =-p_{S} B_{0}^{S} e^{-p_{S}(1-a)}+\tilde{B}_{S} \tilde{L}_{n}^{(m)}\left(p_{S}(1-a)\right) \\
& \eta^{2}: \frac{a}{1-a}\left(p_{L}+\frac{1}{1-a}\right) A_{0}^{L} e^{-p_{L}(1-a)} \\
& -(1-a) p_{L}^{2} B_{0}^{L} e^{-p_{L}(1-a)}+\tilde{A}_{L}\left[-\frac{n}{m+1} p_{L} \tilde{T}_{n m}(x)\right. \\
& \left.+a p_{L} \frac{\partial \tilde{\Psi}_{n m}(x)}{\partial x}\right]_{x=p_{L}(1-a)} L_{n}^{(m)}(0) \\
& +\tilde{B}_{L}\left[\frac{n+m+1}{m+1} p_{L} \tilde{L}_{n}^{(m)}(x)\right. \\
& \left.+a p_{L} \frac{\partial \tilde{L}_{n}^{(m)}(x)}{\partial x}\right]_{x=p_{L}(1-a)} \\
& =-(1-a) p_{S}^{2} B_{0}^{S} e^{-p_{S}(1-a)}+\tilde{B}_{S}\left[\frac{n+m+1}{m+1}\right. \\
& \left.\times p_{S} \tilde{L}_{n}^{(m)}(x)+a p_{S} \frac{\partial \tilde{L}_{n}^{(m)}(x)}{\partial x}\right]_{x=p_{S}(1-a)}
\end{aligned}
$$

Introducing the balance of heat flux at the tip it becomes.

$$
\begin{aligned}
& -\left\{\frac{1}{1-a}\left(p_{L}+\frac{1}{1-a}\right) A_{0}^{L} e^{-p_{L}(1-a)}+p_{L}^{2} B_{0}^{L} e^{-p_{L}(1-a)}\right. \\
& +\left[\tilde{A}_{L} p_{L} \frac{\partial \tilde{\Psi}_{n m}(x)}{\partial x} L_{n}^{(m)}(0)+\tilde{B}_{L} p_{L} \frac{\partial \tilde{L}_{n}^{(m)}(x)}{\partial x}\right]_{x=p_{L}(1-a)} \\
& \left.\quad+\frac{K_{S}}{K_{L}}\left[p_{S}^{2} B_{0}^{S} e^{-p_{S}(1-a)}+\tilde{B}_{S} p_{S} \frac{\partial \tilde{L}_{n}^{(m)}(x)}{\partial x}\right]_{x=p_{S}(1-a)}\right\}=0
\end{aligned}
$$


If the mass flux balance of solute at the tip is taken into account, it can be written as,

$$
\begin{aligned}
p(1 & -k)\left\{-\frac{1}{1-a} A_{0} e^{-p(1-a)}-p B_{0} e^{-p(1-a)}\right. \\
& \left.+\tilde{A} \tilde{\Psi}_{n m}(p(1-a)) L_{n}^{(m)}(0)+\tilde{B} \tilde{L}_{n}^{(m)}(p(1-a))\right\} \\
= & -\left\{\frac{1}{1-a}\left(p+\frac{1}{1-a}\right) A_{0} e^{-p(1-a)}+p^{2} B_{0} e^{-p(1-a)}\right. \\
& \left.+\left[\tilde{A} p \frac{\partial \tilde{\Psi}_{n m}^{(x)}}{\partial x} L_{n}^{(m)}(0)+\tilde{B} p \frac{\partial \tilde{L}_{n}^{(m)}(x)}{\partial x}\right]_{x=p(1-a)}\right\}
\end{aligned}
$$

From the relation between temperature and solute on the interface, Eqs. (20) and (21) are obtained.

$$
\begin{aligned}
\eta^{0}:- & p B_{0}^{S} e^{-p_{S}(1-a)}+\tilde{B}_{S} \tilde{L}_{n}^{(m)}\left(p_{S}(1-a)\right) \\
= & m_{0}\left\{-\frac{1}{1-a} A_{0} e^{-p(1-a)}-p B_{0} e^{-p(1-a)}\right. \\
& \left.+\tilde{A}_{n m}(p(1-a)) L_{n}^{(m)}(0)+\tilde{B} \tilde{L}_{n}^{(m)}(p(1-a))\right\} \\
\eta^{2}: \quad- & (1-a) p_{S}^{2} B_{0}^{S} e^{-p_{S}(1-a)}+\tilde{B}_{S}\left[\frac{n+m+1}{m+1} p_{S} \tilde{L}_{n}^{(m)}(x)\right. \\
& \left.+a p_{S} \frac{\partial \tilde{L}_{n}^{(m)}(x)}{\partial x}\right]_{x=p_{S}(1-a)} \\
= & \frac{\gamma}{\Delta S} \frac{2}{\rho}+m_{0}\left\{\frac{a}{1-a}\left(p+\frac{1}{1-a}\right) A_{0} e^{-p(1-a)}\right. \\
& -(1-a) p^{2} B_{0} e^{-p(1-a)}+\tilde{A}\left[-\frac{n}{m+1} p \tilde{\Psi}_{n m}(x)\right. \\
& \left.+a p \frac{\partial \tilde{\Psi}_{n m}(x)}{\partial x}\right]_{x=p(1-a)} L_{n}^{(m)}(0) \\
& \left.+\tilde{B}\left[\frac{n+m+1}{m+1} p \tilde{L}_{n}^{(m)}(x)+a p \frac{\partial \tilde{L}_{n}^{(m)}(x)}{\partial x}\right]_{x=p(1-a)}\right\}
\end{aligned}
$$

The solutions of the linear relations among these parameters can easily be obtained by the same way as used in Reference 6). At first Eqs. (9) (14) and (16) (21) are solved, and the parameters are determined as functions of the radius of dendrite tip curvature, $\rho$. Secondarily, the value of $\rho$ is determined so as to satisfy Eq. (15). By this way it is possible to predict the radius of curvature for the dendrite tip which is corresponding to the solidification conditions.

\section{Application of the Theory for Dendrite Growth}

The temperature and the solute distribution in liquid around solid can be determined by the analytical model stated above, because all parameters for the fields are determined. By applying the solute conservation in the area perpendicular to the growth direction, it is possible to predict the primary arm spacing of the array of dendrite. The prediction is done by the following way.

If it is assumed that the secondary arm of a dendrite can grow as far as the relation given in Eq. (22) is satisfied, the following relation is realized.

$$
T_{L}(\xi, \eta) \leqq T_{M}+\frac{\gamma}{\Delta S} K(\xi, \eta)+m_{0} C_{L}(\xi, \eta)
$$

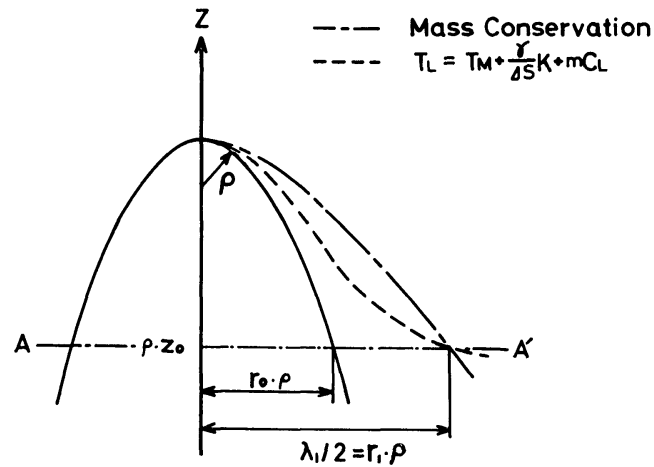

Fig. 1. Schematic representation of the solute conservation.

This restricts the possible region where the secondary arm can grow.

On the other hand, the solute conservation on the plane $A-A^{\prime}$ shown in Fig. 1 is given as

$$
\int_{0}^{r_{0}} k C_{L}\left(\xi^{2}=1\right) \cdot 2 \pi r d r+\int_{r_{0}}^{r_{1}} C_{L}\left(z=z_{0}\right) \cdot 2 \pi r d r=C_{\infty}^{L} \cdot \pi r_{1}^{2}
$$

The points satisfying both Eqs. (22) and (23) define an extreme area where the secondary arm can grow and satisfy the condition of solute conservation around the dendrite. From the points, the primary arm spacing can be defined as

$$
\lambda_{1}=2 \rho r_{1}
$$

\section{Comparison with Experimental Results and Discussion}

The radii of curvature and the tip temperatures for $\mathrm{Al}-4.0$ mass $\% \mathrm{Cu}$ alloy predicted by using different values of the shape parameter " $a$ " are shown in Figs. 2 to 4 . The constants used in this analysis are listed in Table 1. Figure 2 shows the relations between the predicted radius of tip curvature and the growth rate calculated with different values of shape parameter $a(a=0.3$ corresponds to hyperbolic tip and $a$ for $B_{0}^{L}=0$ (Fig. 4) corresponds to elliptic one). The radius predicted for $a=0$ and that for $a$ for $B_{0}^{L}=0$ are very close with each other. Figure 3 shows the relation between the tip temperature predicted and the growth rate. The effects of shape parameter on the tip temperature are larger than those on the tip radius. From Figs. 2 and 3, it can be seen that the the effect of the shape parameter of the interface on the radius of curvature is small. This means that the elliptic tip approximation for real dendrite is rather good for dimensions, but not good for tip temperature.

Trivedi ${ }^{4)}$ proposed the model based on the hemispherical tip. In this model, the temperature field in liquid is given as

$$
T_{L}=T_{t}^{L}+\rho G_{L}\left(z-\frac{1}{2}\right)+U_{L}(\xi, \eta)
$$

where $U_{L}$ satisfies the diffusion equation. The temperature field in solid and the solute concentration 
Table 1. Parameters used in the calculation for Al-Cu alloy.

\begin{tabular}{c|cccccccc}
\hline Parameter & $m_{0}$ & $k$ & $D$ & $\gamma / \Delta S$ & $\Delta H / c_{1}$ & $K_{S} / K_{L}$ & $C_{\infty}^{L}$ & $G_{L}$ \\
\hline Value & $-2.5 \mathrm{~K}(\mathrm{mass} \%)^{-1}$ & 0.14 & $5.0 \times 10^{-9} \mathrm{~m}^{2} \mathrm{~s}^{-1}$ & $1.04 \times 10^{-7} \mathrm{mK}$ & $447 \mathrm{~K}$ & 2.33 & $4.0 \mathrm{mass}^{2}$ & $40 \times 10^{2} \mathrm{Km}^{-1}$ \\
\hline
\end{tabular}

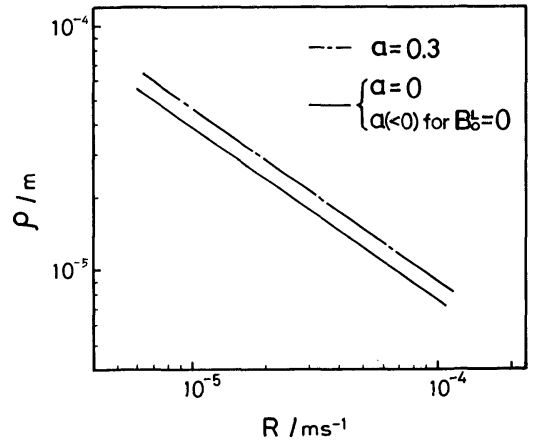

Fig. 2. Variation of the radius of curvature with the growth rate for different shape parameter " $a$ ". The radius of curvature corresponding to $a$ in Fig. 4 is very close and a little smaller than that of $a=0$.

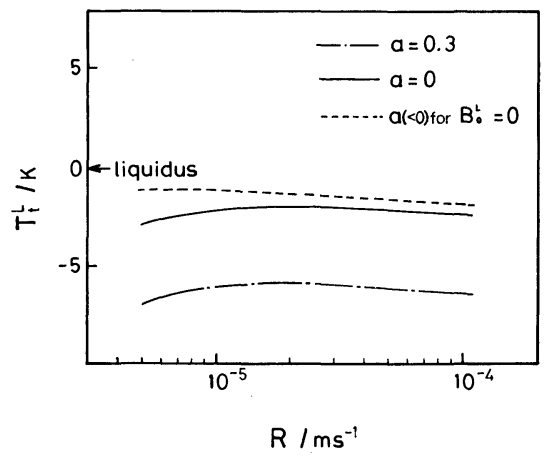

Fig. 3. Variation of the tip temperature with the growth rate for different shape parameter " $a$ ".

field are also calculated in the same manner as mentioned above. The term $G_{L}(z-0.5)$ is introduced in order to satisfy the unidirectional solidification condition, but it does not necessarily satisfy the diffusion equation. Moreover, the perturbative stability condition of hemispherical tip is assumed in the model, and a free parameter, which is given empirically, is introduced in the model.

In the model used in the current work, the term corresponding to $G_{L}(z-0.5)$ is given by

$$
B_{0}^{L} e^{-p_{L} \xi^{2}} e^{p_{L} \eta^{2}}=B_{0}^{L} e^{-2 p_{L} z}
$$

and it gives the same asymptotic behaviour for small $z$, that is the region close to the tip. This term is also a solution of the diffusion equation, and behaves like a solution for planar interface. So it behaves as the averaged field for arrayed dendrites. This field is responsible for the piling up of solute in the liquid along the interface. The value of " $a$ " is shown in Fig. 4, in the case that the field gives no contribution. The perturbative stability condition is introduced as a result that the interface is also under the local equilibrium when the perturbative deformation is applied to the interface. Then the model has no free parameter.

The predicted primary arm spacing predicted with $a=0$ for Fe-C alloy is shown in Figs. 5 and 6, where

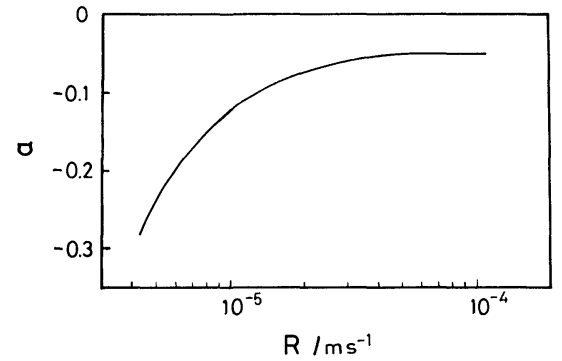

Fig. 4. Value of shape parameter $a$ for $B_{0}^{L}=0$.

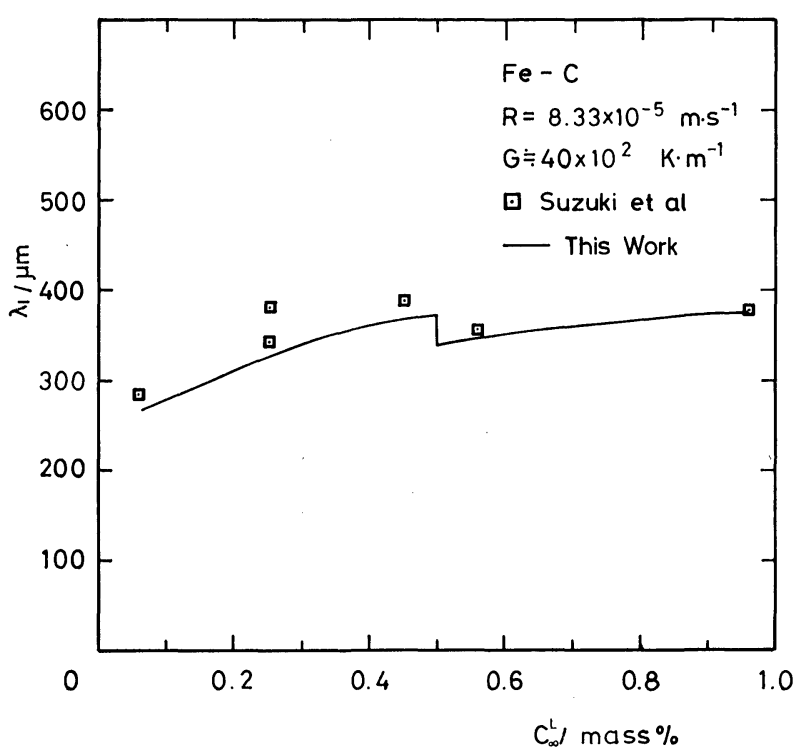

Fig. 5. Variation of the primary arm spacing with the initial concentration of solute $\left(G_{L}=40 \times 10^{2} \mathrm{Km}^{-1}\right)$.

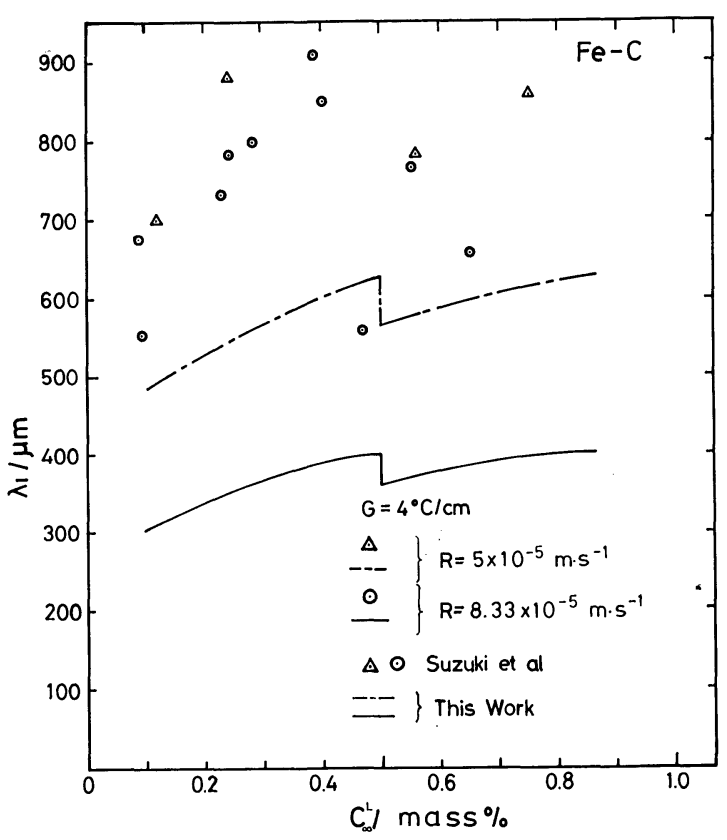

Fig. 6. Variation of the primary arm spacing with the initial concentration of solute $\left(G_{L}=4 \times 10^{2} \mathrm{Km}^{-1}\right)$. 
Table 2. Parameters used in the calculation for $\mathrm{Fe}-\mathrm{G}$ alloy.

\begin{tabular}{l|l|l}
\hline Parameter & \multicolumn{1}{|c|}{ Value for $\delta$} & \multicolumn{1}{|c}{ Value for $\gamma$} \\
\hline$m_{0}$ & $-81 \mathrm{~K}(\operatorname{mass} \%)^{-1}$ & $-65 \mathrm{~K}(\operatorname{mass} \%)^{-1}$ \\
$k$ & 0.17 & 0.35 \\
$D$ & $2.0 \times 10^{-8} \mathrm{~m}^{2} \mathrm{~s}^{-1}$ & $2.0 \times 10^{-8} \mathrm{~m}^{2} \mathrm{~s}^{-1}$ \\
$\gamma / \Delta S$ & $1.90 \times 10^{-7} \mathrm{mK}$ & $1.90 \times 10^{-7} \mathrm{mK}$ \\
$\Delta H / c_{1}$ & $348 \mathrm{~K}$ & $348 \mathrm{~K}$ \\
$K_{S} / K_{L}$ & 2.0 & 2.0 \\
\hline
\end{tabular}

the experimental data ${ }^{7)}$ are also shown for comparison. The constants used in this analysis are listed in Table 2.

The primary arm spacing of $\mathrm{Fe}-\mathrm{C}$ alloy predicted shows a very good correspondence to dependence on the solute concentration irrespective of $\delta$ or $\gamma$ of the primary phase as shown in Fig. 5. For the dependence at low temperature gradient the prediction gives smaller value than the experimental one as shown in Fig. 6. Under the condition of low temperature gradient, the primary arm spacing becomes larger than that for higher temperature gradient, and the temperature gradient in the experiment is given as the averaged one in the area including the interdendritic region. The definition of temperature gradient at the tip in this text is not so adequate for the approximation of real temperature gradient, because the contribution of the temperature gradient in the interdendritic region to the averaged tempera ture gradient is larger than that in higher temperature gradient. Instead of the temperature gradient at the dendrite tip defined by Eq. (12), the temperature gradient in the interdendritic region in the liquid is defined as Eq. (27).

$$
\tilde{G}_{L}=-\frac{2}{\rho} p_{L} B_{0}^{L} e^{-p_{L}}
$$

Figure 7 shows the primary arm spacing predicted under the new definition. The prediction for low temperature gradient shows better agreement with the experimental result than that calculated by the previous definition of the temperature gradient.

Using the models previously reported, the radius of tip curvature and the primary arm spacing were determined under the condition of solute conservation in the area perpendicular to the growth direction. Since the primary arm spacing can not be obtained under this condition without adopting a certain assumption, one must give the plane where the conservation law is applied to. Hunt ${ }^{8)}$ assumed this plane as the one with the solidus temperature. In the model proposed in this paper, the quadratic plane satisfying the condition shown below is adopted.

$$
T_{L}=T_{M}+\frac{\gamma}{\Delta S} K+m_{0} C_{L}
$$

It is necessary to make further experimental and theoretical studies in order to clarify whether or not the plane should be adopted for the prediction to

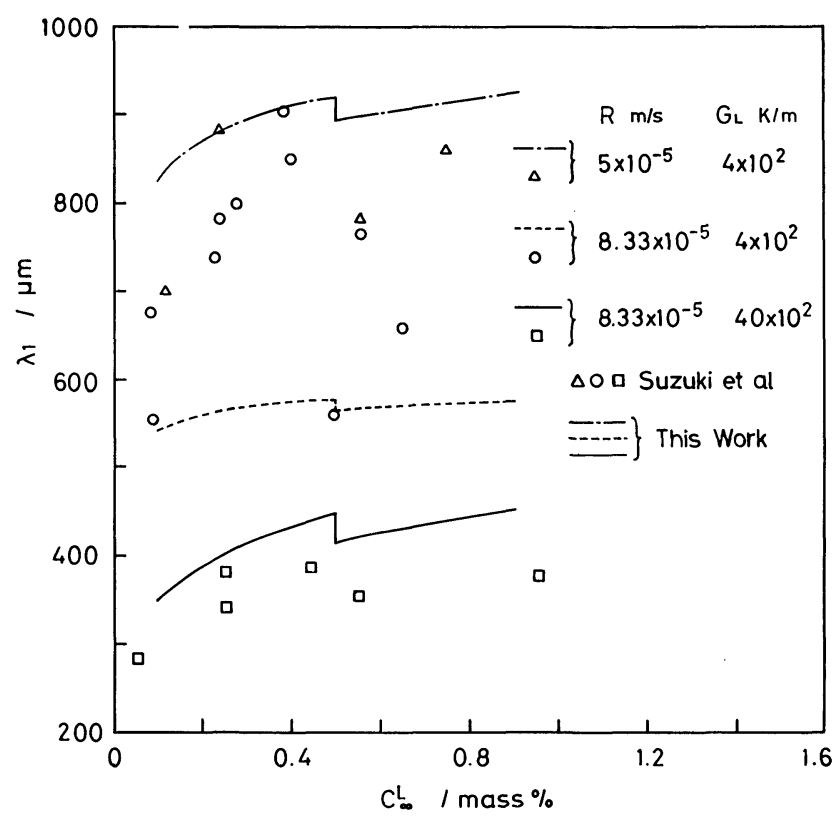

Fig. 7. Variation of the primary arm spacing with the initial concentration of solute. Temperature gradient $\tilde{G}_{L}$ is defined in the interdendritic region (Eq. (27)).

primary arm spacing.

\section{Conclusions}

The shape effect of dendrite tip on the dimensions of dendrite is studied on the basis of the perturbation theory for the dendrite growth. A model for the prediction of primary arm spacing is also proposed to show the applicability of the perturbation theory for the dendrite growth.

The shape effect on the radius of dendrite tip curvature is rather small, and this is the reason why the models based on the elliptic tip of dendrite give rather good predictions for the dimensions of dendrite.

The primary arm spacing predicted agreed fairly well with the experimental ones with respect to the dependence on the solute concentration. For low temperature gradient it is pointed out that ambiguity still remains on the definition of the real temperature gradient in the theory.

\section{Nomenclature}

$a$ : shape parameter of the interface $A_{0}, A_{0}^{L}, \tilde{A}, \tilde{A}_{L}:$ $\left.\begin{array}{l}A_{0}, A_{0}^{L}, A, \tilde{A}_{L}: \\ B_{0}, B_{0}^{L}, B_{0}^{S}, \tilde{B}, \tilde{B}_{L}, \tilde{B}_{S}:\end{array}\right\}$ parameters to be determined

$c_{1}$ : heat capacity

$C_{L}$ : concentration of solute in the liquid

$C_{\infty}^{L}$ : the initial concentration of solute

$E_{1}(x)$ : integral exponential function .

$G_{L}:$ temperature gradient

$\Delta H:$ latent heat

$k$ : equilibrium distribution coefficient

$K$ : curvature of the interface

$K_{L}, K_{S}$ : thermal conductivities of liquid and solid

$L_{n}^{(m)}(x)$ : Laguerre polynomial

$\tilde{L}_{n}^{(m)}(x)=e^{-x} x^{m / 2} L_{n}^{(m)}(x)$

$m_{0}$ : liquidus slope 
$m, n:$ non-negative integers

$p_{L}, p_{S}$ : Peclet numbers for thermal diffusion in liquid and in solid,

$p$ : Peclet number for diffusion of solute in liquid

$\Delta S$ : entropy of fusion per unit volume

$\mathcal{T}_{L}, T_{S}:$ temperatures of the liquid and of the solid

$T_{0}^{L}, T_{0}^{S}:$ parameters to be determined

$T_{t}^{L}:$ tip temperature

$T_{M}$ : melting temperature

$(x, y, z):$ dimensionless Cartesian coordinate

$(\xi, \eta, \phi)$ : dimensionless parabolic coordinate

$\Delta \xi^{2}$ : perturbation of the interface

$\rho:$ radius of curvature

$\lambda_{1}$ : primary arm spacing

$\gamma:$ interfacial free energy

$\delta_{n m}:$ parameter

$\Phi_{n m}(x)$ : confluent hypergeometric polynomial of first kind, $\Phi(n+m+1, m+1 ; x)$

$\Psi_{n m}(x)$ : confluent hypergeometric polynomial of second kind, $\Psi(n+m+1, m+1 ; x)$

$$
\tilde{\Psi}_{n m}(x)=e^{-x} x^{m / 2} \Psi_{n m}(x)
$$

\section{Acknowledgements}

The authors wish to express their sincere thanks to Professor Dr. T. Umeda, the University of Tokyo for his valuable discussions.

\section{REFERENCES}

1) W. Kurz and D. J. Fisher: Acta Met., 29 (1981), 11.

2) W. W. Mullins and R. F. Sekerka: J. Appl. Phys., 35 (1964), $328 \& 444$

3) M.H. Burden and J. D. Hunt: J. Crystal Growth, 22 (1974), 109.

4) R. Trivedi: J. Crystal Growth, 49 (1980), 219.

5) J.S. Langer and H. Müller-Krumbhaar: Acta Met., 26 (1978), 1681

6) Y. Miyata and T. Suzuki: Met. Trans., 16A (1985), 1807.

7) M. Suzuki, T. Kitagawa and S. Miyahara: Tetsu-toHagané, 71 (1985), S1034.

8) J. D. Hunt: Solidification and Casting of Metals, The Metal Soc., London, (1979), 3. 